\title{
A small case series about safety and effectiveness of a hypofractionated electron beam radiotherapy schedule in five fractions for facial non melanoma skin cancer among frail and elderly patients
}

\author{
Gianluca Ferini ${ }^{1}$, Laura Molino ${ }^{2}$, Laura Bottalico ${ }^{3}$, Paolino De Lucia ${ }^{3}$, Francesco Garofalo ${ }^{3}$ \\ ${ }^{1}$ REM Radioterapia srl, Viagrande (CT), Italy \\ ${ }^{2}$ Radiation Oncology Unit - Department of Biomedical, Dental Science and Morphological and Functional Images, \\ University of Messina, Messina, Italy \\ ${ }^{3}$ Malzoni Radiosurgery Center, Agropoli (SA), Italy
}

\begin{abstract}
Background: The aim of the study was to evaluate local control and toxicities of strongly hypofractionated electron beam radiotherapy (RT) in elderly and fragile patients with facial nonmelanoma skin cancer (NMSC).

Materials and methods: We enrolled patients aged $\geq 65$ years with facial NMSC, Karnofsky Performance Status (KPS) $\geq 40$ and life expectancy $\geq 6$ months, amenable neither to daily RT nor surgery. Radiotherapy consisted of 35 Gy, delivered with $6 \mathrm{MeV}$ electron beam, in 5 fractions of $7 \mathrm{~Gy} /$ day twice a week (tw). Prescription isodoses were $100 \%$ for cT1-cT2 and $90 \%$ for cT3-cT4. Objective response was assessed clinically 4 and 8 weeks after the end of RT and then monitored every 6 months. Side effects were assessed according to the CTCAE scale.

Results: 12 patients of median age 89.5 years with a total of 23 NMSC cN0 achieved a median follow-up time of 6 months (range 1-10), with total treatment compliance. 10/12 patients had a $40 \leq$ KPS $<70$ and $2 / 12$ a $70 \leq$ KPS $<90.5 / 12$ patients had synchronous lesions. 22/23 lesions were classified as T1-T2 and had complete response (CR), 1/23 as T4 with partial response (PR). Within 4 weeks after the end of treatment, G1 toxicity was reported for $12 / 23$ lesions, G2 for 8/23, G3 for 3/23, G4 for 0/23, all disappeared 8 weeks later, with or without topical therapy. After last follow-up (1 June 2020) 1/12 patients died with PR from senile marasmus, $11 / 12$ are alive with CR and widely tolerated toxicities.

Conclusions: Extreme hypofractionation of radiotherapy dose for facial NMSC is effective, safe and suitable for elderly patients. Key words: NMSC; electron beam radiotherapy; elderly patients; hypofractionated radiotherapy; skin cancer; brachytherapy Rep Pract Oncol Radiother 2021;26(1):66-72
\end{abstract}

\section{Introduction}

Non-melanoma skin cancers (NMSC), in the basal cell and squamous cell variants, represent the most frequent tumor pathology of the skin, with a clear prevalence of the first histotype over the second, as suggested by some European and
American registries [1-5]. All these epidemiological sources agree in indicating the advanced age, the white skin phototype and the prolonged sun exposure, especially related to specific occupational activities, as the main risk factors for the development of these tumors. The damage induced by ultraviolet radiation is cumulative so the risk of 
developing NMSC becomes more pronounced at a very advanced age, a biological era in which, due to the presence of comorbidity or poor compliance of the patient, the range of therapeutic choices that normally includes surgical excision, radiotherapy, electrocoagulation, cryotherapy, photodynamic and topical therapies (ie 5-fluorouracil, imiquimod, etc.), vismodegib (for basal cell carcinomas), is restricted to the exclusive use of some of them, often in palliative settings. Radiation therapy (RT) emerges among the most comfortable therapeutic options and guarantees good disease control even in schedules with dose hypofractionation. Furthermore, radiotherapy is offered as a more effective and safe alternative to surgery in those cases where lesions are on surgically inaccessible sites or which involve both cosmetic and functional impairments (pinna, nasal pyramid, vermillion border, angulus oculi medialis, etc.). We report here the toxicity and local disease control results of a small series of elderly patients with NMSC of the face, not suitable for surgical treatment either by comorbidity or by functional outcome or by patient choice, treated with radiotherapy employing a non-standard dose hypofractionation scheme.

\section{Materials and methods}

Patients not amenable to daily treatment, aged over 65 years and with histological confirmation of basal cell (BCC) or squamous cell carcinoma (SCC) of the face, were considered as candidates for this treatment. These patients were preliminarily examined by radiation oncologists and plastic surgeons. Patients excluded from surgical treatment for comorbidities, multifocal lesions with problematic reconstruction, frailty or by their own choice were referred to RT. Given the short duration of the radiotherapy schedule, for better patient compliance, minimum eligibility criteria for treatment were a Karnofsky Performance Status (KPS) of at least 40 and a life expectancy of at least 6 months. In cases of complex morphology or clinically suspected lesions due to deep infiltration (T3, T4 according to TNM staging system), at least one confirmation CT scan was required to guide the choice of the isodose to which the $6 \mathrm{MeV}$ Electron energy treatment dose was prescribed: $90 \%$ in the case of deeper lesions, $100 \%$ in the case of more superficial lesions.
The electron applicator mounted to the gantry head was positioned in contact with the skin so that source to skin distance (SSD) and source to collimator distance (SCD) were both equal to 100 $\mathrm{cm}$. Collimators of various shapes (circular, quadrangular or rectangular) and size were used looking for an expansion of at least $1.5-2 \mathrm{~cm}$ around the macroscopic disease in order to compensate for the large field edge penumbra region, characteristic of electrons, and cover the subclinical disease. It was decided not to use bolus due to the difficult application in areas with poor adherence. In lesion sites near the eye (nasal root, inner canthus of the eye, cheekbone) where it is not possible to position the applicator in contact with the skin, we used lead cutout to shield it from the lateral scattering of the electron beam.

Patients were immobilized using a thermoplastic mask, suitably cut to expose the skin area to be treated. The radiotherapy treatment, consisting of 5 fractions of $7 \mathrm{~Gy}$ each, was delivered every Monday and Thursday or, alternatively, every Tuesday and Friday, so that it was completed in 15 days. The follow-up was the time elapsed from the starting date of radiotherapy to the date of the last analysis (June 1, 2020). Local recurrence-free survival was determined by the Kaplan-Meier method. The objective response was evaluated clinically 4 and 8 weeks after the end of the treatment and every 6 months thereafter and photographically recorded. The side effects were assessed and noted using the Common Terminology Criteria for Adverse Events (CTCAE). Obtaining informed consent was mandatory for processing.

\section{Results}

In the period between 1 August 2019 and 1 June 2020 we enrolled 12 patients (8 males, 4 females) of median age 89.5 years (range 66-98 years) with a total of 23 localizations of NMSC $\mathrm{cN} 0$ (on average 1.9 lesions/patient), including 8 squamous cell carcinomas and 15 basal cell carcinomas (Tab. 1). The median follow-up time was 6 (range 1-10). Compliance with treatment was excellent, with all patients having completed the schedule. Only 4/23 lesions had previously been removed and judged not to be further surgically attackable at recurrence; all the other ones had not received any previous treatment. 6/12 patients lived in nursing homes for elderly people 
Table 1. Patients' characteristics

\begin{tabular}{|c|c|}
\hline Median age (years) & $89.5(66-98)$ \\
\hline \multicolumn{2}{|l|}{ Gender } \\
\hline Female & $4(33.3 \%)$ \\
\hline Male & $8(66.7 \%)$ \\
\hline \multicolumn{2}{|l|}{ Histotype } \\
\hline Squamous cell carcinoma & $8(34.8 \%)$ \\
\hline Basal cell carcinoma & $15(65.2 \%)$ \\
\hline \multicolumn{2}{|l|}{ Stage } \\
\hline T1 tumors (clinically evaluated) & $9(39.1 \%)$ \\
\hline T2 tumors (clinically evaluated) & $13(56.6 \%)$ \\
\hline T4 tumors (radiologically confirmed) & $1(4.3 \%)$ \\
\hline \multicolumn{2}{|l|}{ Tumor site } \\
\hline Nose & $2(8.7 \%)$ \\
\hline Zygomatic area & $5(21.7 \%)$ \\
\hline Eyelid-periorbital area & $1(4.3 \%)$ \\
\hline Ear, pre- and retroauricolar region & $5(21.7 \%)$ \\
\hline Cheek & $5(21.7 \%)$ \\
\hline Forehead-temples & $3(13 \%)$ \\
\hline Vertex & $2(8.7 \%)$ \\
\hline \multicolumn{2}{|l|}{ Acute skin toxicity sec. CTCAE } \\
\hline G1 & $12(52.2 \%)$ \\
\hline G2 & $8(34.8 \%)$ \\
\hline G3 & $3(13 \%)$ \\
\hline G4 & 0 \\
\hline \multicolumn{2}{|l|}{ Response } \\
\hline Complete responses & $22(95.7 \%)$ \\
\hline Partial responses & $1(4.3 \%)$ \\
\hline
\end{tabular}

CTCAE - Common Terminology Criteria for Adverse Events

or needed home health care, $4 / 12$ presented health problems that severely limited their autonomy in daily activities (10/12 patients with $40 \leq \mathrm{KPS}<70)$, $2 / 12$ were fairly autonomous ( $70 \leq \mathrm{KPS}<90) .5 / 12$ patients had synchronous lesions.

8/12 patients had cardiovascular and/or dysmetabolic and/or neurodegenerative diseases in their medical history. 22/23 lesions were classifiable as T1-T2 $(<5 \mathrm{~cm}), 1 / 23$ as T4. 22/23 lesions had a complete documented clinical response to the 4 weeks or 8 weeks scheduled physical exam. 1/23 had a partial response, a voluminous squamous cell carcinoma cT4 with dimensions over $8 \mathrm{~cm}$ in diameter involving almost half of the face with extensive invasion of the underlying tissues, bleeding enough to condition the onset of a moderate-severe anemia $(8 \mathrm{~g} / \mathrm{dL})$ in which the therapeutic goal was to ob- tain a lasting hemostasis, effectively achieved and documented at the first post-treatment check. At the end of the radiotherapy treatment and/or at the control at 4 weeks 12/23 lesions reported a G1 toxicity, 8/23 G2, 3/23 G3, no G4 toxicity. All of them, however, regressed at the control after 8 weeks, with or without topical therapy, and also not showing any correlation between the experienced grade of toxicity, the location and the tumor stage (Fig. 1,2). At the date of the last follow-up (1 June 2020) 1/12 died due to senile marasmus with a partial clinical response, 11/12 are alive with a complete clinical response and no lasting toxicities that could compromise the patient cosmetic satisfaction. The actuarial rate of local control with complete clinical response at 6 months was $95.5 \%$, corresponding to a progression-free survival at 6 months of $100 \%$. There was no cancer-related death.

\section{Discussion}

Radiation therapy in the treatment of skin cancers can be applied, as well known, through various methods that include KV X-rays, high energy photons (4-6 MV), brachytherapy and external electron beam. Cognetta et al. [6], in a broad retrospective analysis of 1715 NMSC, reported excellent results both in terms of local control at 2 and 5 years (relapse rates of $1.9 \%$ and $5 \%$, respectively) and cosmetic results (from good to excellent in all cases) with a fractionation scheme of $35 \mathrm{~Gy}$ in 5 fractions of 7 Gy/day delivered using superficial X-rays that offered treatment success rates and functional outcomes comparable to those achievable with Mohs surgery. The kilo- and orthovoltage units (50-300 KV) generally do not require bolus for a dose targeted to superficial tissues. Conversely, superficial X-rays are not ideal to treat skin lesions $\geq \mathrm{T} 3$ according to TNM staging system. The greater versatility of the high energy photons (4-6 MV) produced by the linear accelerator (LINAC), integrated with bolus of variable thickness for the modulation of the dose in depth and carefully monitored by the modern Treatment Planning System lead to the progressive abandonment of the kiloand orthovoltage X-ray treatment units. However, this choice is more cumbersome, time-consuming and often worse than the other methods; therefore, it is strongly discouraged in the cases of lesions localized in anatomical areas to which, due to their 


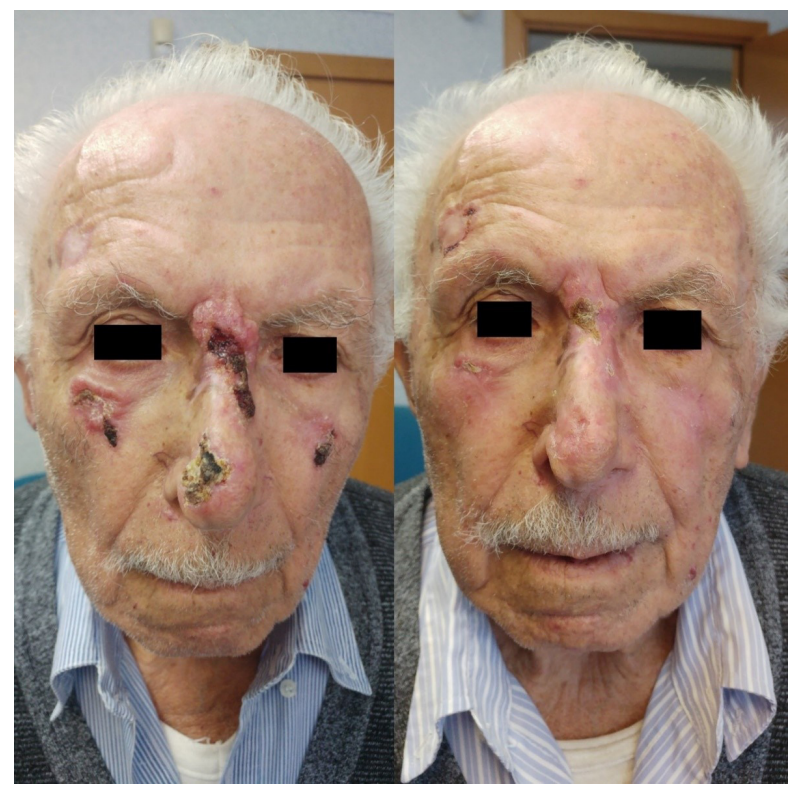

Figure 1. A patient with multiple facial non-melanoma skin cancer (NMSC): on the left pre-radiotherapy status, on the right -8 weeks after RT status

conformation, it is hard to apply a bolus adhering to the target surface (i.e. nasal root, inner canthus of the eye, lower lid crease, eyelid cheek junction, etc.), that is required for a correct distribution of the prescribed dose.

Modern gamma-emitting devices for contact and/or interstitial brachytherapy overcome these limits, guaranteeing better adhesion to irregular or curved surfaces and also benefit from a steeper dose fall off that helps to save surrounding healthy tissues and to achieve functional and cosmetic result (good to excellent between 78 and $92 \%$ of cases) $[7,8]$. This method requires particular skills, is not so widespread and relies on availability of a radioisotope whose efficiency decays naturally over time. All these factors limit therefore its choice by most radiation oncologists.

In this context, the use of electrons emitted from LINAC is a choice that is easily available, effective and with an optimal dose distribution profile. The low energy electron beams $(3-4 \mathrm{MeV})$, indeed, have a short build-up (between 5 and $10 \mathrm{~mm}$ ) with a release of $80 \%$ of the dose already on the surface, thus being able to avoid the application of a bolus, and with a low diffusion to the underlying tissues such as subcutaneous fat, muscles and bone, thus limiting toxicity. On the other hand, this technique gives a shadowing deposition on the field edges that requires an expansion of at least $1 \mathrm{~cm}$ for the

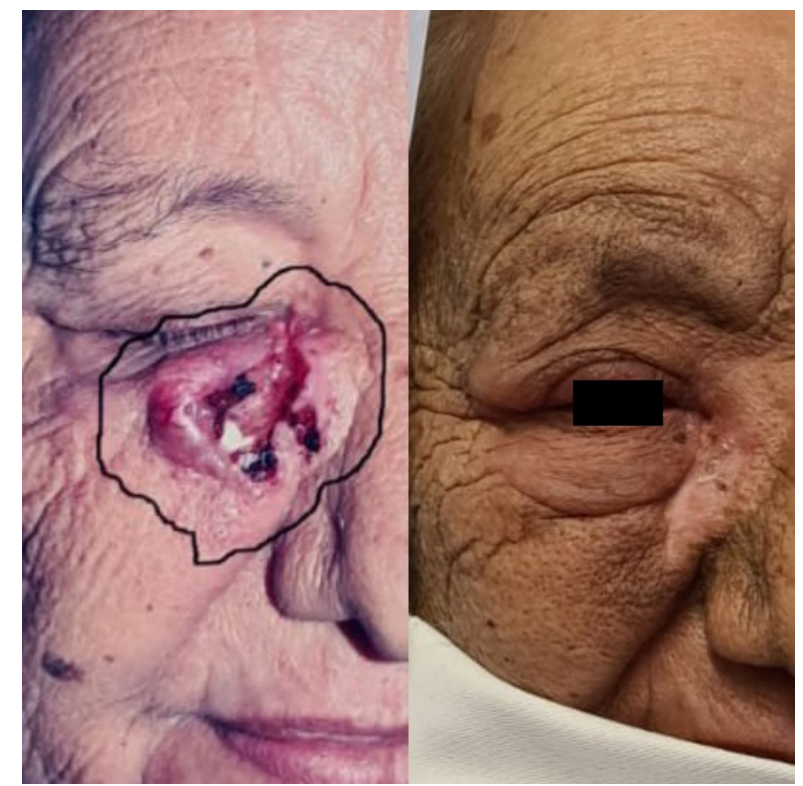

Figure 2. A patient with a non-melanoma skin cancer (NMSC) lesion localized at the inner canthus of the right eye: on the left pre-radiotherapy status, on the right -8 weeks after RT status

optimal coverage of the clinical target, driven by the final collimation in contact with the skin. These considerations motivate our clinical practice as well as that of most radiation oncologists who do not have the above techniques and are supported by works such as those of Scholten et al. [9]. The recent overview by Pashazadeh et al. [10] examines the pros and cons of each method and, although promoting the topical application of beta-emitters capable of maximizing the dose to the tumor while minimizing that to healthy tissue, recognizes its limited availability and suggests the electrons produced by LINAC as a valid alternative.

Concerning hypofractionation, works such as those of van Hezewijk and Kwan et al. [11, 12] underline its safety and efficacy, having obtained in their experiences (two-arms and multi-regimen, respectively) local control rates and cosmetic results comparable to those achievable with 2-3 Gy/fraction regimes by using doses per fraction of $4.4 \mathrm{~Gy}$ and 7 Gy, respectively. Similarly, Pelissero et al. and the subsequent update by Russi et al. have reported long-lasting complete response in almost $100 \%$ of elderly patients (median age 82.5 years) with 159 BCC of the face T1-T2 treated with an exclusive radiotherapy schedule of 25-30 Gy in 5-6 fractions of 5 Gy once a week, with acute skin toxicity not higher than level 2 in only $30 \%$ of cases $[22,23]$. 
However, since our clinical records also included cases of squamous cell carcinoma, we believed that the above dose could be insufficient, especially for more extended tumors.

The survey produced in 2014 in the UK highlighted the extreme heterogeneity of the fractionation schedules used in different radiotherapy centers, reporting the following as the most frequent: 18-20 Gy/1 fx, 35 Gy/5 fx, 45 Gy/10 fx and 55 Gy/20 fx [13]. The single fraction recognizes as a limit of applicability the tumor extension which must be less than $3 \mathrm{~cm}$ [14] while the last two schedules require a number of accesses that may not satisfy the compliance of the elderly patient. For such reasons, we believe that the dose of 35 Gy in 5 fractions of 7 Gy/day can represent an adequate therapeutic option; the bi-weekly schedule is supported by the tables of the time dose fractionation factor by Orton and Ellis, from which, for the examined dose, it is possible to extrapolate a therapeutic index equal to 98 , favorably between the suggested limits of 90-110 [15].

Furthermore, assuming an alpha/beta ratio equal to 14 for the tumor, this fractionation allows the achievement of a biologically effective dose (BED) of $52.5 \mathrm{~Gy}$, adequate for disease control. On the other hand, such a measure will correspond to 116.67 Gy for healthy tissues, for which the $\alpha / \beta$ ratio is assumed equal to 3 , that is sufficiently safe in terms of late toxicity. Indeed a hazard ratio for local relapse significantly greater than 1 has been reported e.g. by Silva et al. [16] upon failure to achieve the aforementioned tumor BED threshold and late sequelae were observed in less than $6 \%$ of cases irradiated with $35 \mathrm{~Gy} / 5 \mathrm{fx}$, results considered acceptable by us. The review by Zaorsky et al. [18] is in agreement with these results, mentioning the prescription dose of $35 \mathrm{~Gy}$ in $5 \mathrm{fx}$ among the schedules which, not deviating excessively from a $\mathrm{BED}_{3} \approx 100$ for healthy tissues, guarantees a good cosmetic result in at least $80 \%$ of cases.

Also the recent review by Gunaratne et al. [17] encourages the use of hypofractionation in the frail elderly patient, where the reduction of the overall treatment time is often crucial to comply with prescription directions, and promotes the schedules with 1-3 sessions per week and a dose/fraction of 5-7 Gy for a total dose between 30 and $40 \mathrm{~Gy}$ as more effective and tolerable.

In a fairly broad retrospective analysis performed on 334 NMSC ear lesions irradiated mostly with
orthovoltage-X-rays (in $83 \%$ of cases) or electrons (12\%), adopting the same prescription as that tested in our experience for 123 lesions but hypofractioning in almost all cases, the authors reported a promising 2 and 5 year local control rate of $86.6 \%$ and $79.2 \%$, respectively, with a cancer-specific survival of $96 \%$ at the same time points. Although the use of electrons (together with lesion dimensions, tumor histology, field size and a longer overall duration of treatment) correlated, in a univariate analysis, with a worse local failure rate, this evidence was not confirmed by multivariate analysis, where only tumor size and BED (with a hazard ratio of 1.69 for every 5 Gy decrease) turned out to be significant predictors of the risk of recurrence $(\mathrm{p}=0.02$ and $p=0.03$, respectively). In addition to local control, another reassuring data is the severe toxicity rate (G4 according to the RTOG scale $=$ necrosis and ulceration of the treated area that persist for over 3 months and/or with the need for surgical treatment), found in only $4.9 \%$ of patients at 2 years and in $7.3 \%$ at 5 years and linked to dose hypofraction. This, however, was particularly true for the dose per fraction of 4.3-4.5 Gy (severe late toxicity in $14.5 \%$ of cases) and for the dose per fraction of 7 Gy (5.7\%), while the schedules with dose per fraction $<3$ Gy were not followed by any severe adverse event (0\%) [16].

Just like the ear, the nose could constitute, due to the cartilaginous skeleton on which the integumentary system rests, an anatomical region particularly susceptible to radiation damage with significant cosmetic sequelae in case of hypofractionation of the radiotherapy dose. However, this suspicion is denied in the work of Tsao et al. in which no grade 4 toxicity according to the RTOG scale was detected, not even among patients who had received a dose of $35 \mathrm{~Gy}$ in 5 fractions on consecutive days, against an exceptional disease control (90\% at 2 years) [19]. Our experience relies on the same inspiration as that of Veness [20], and actually investigates the efficacy and tolerability of a strongly hypofractionated radiotherapy scheme. Our results have proved to be in accordance with evidence in literature, although with limited follow-up, and promote as sufficiently safe the use of the scheme adopted by us on a larger elderly and fragile population, especially among the subjects who are not candidates for surgery due to high anesthesiological risk, with multiple synchronous lesions, located in areas where 
the surgical approach would be mutilating and cosmetically unacceptable, or with poor performance status, presence of severe comorbidities, in therapy with anticoagulants or antiplatelet agents, limited life expectancy, hospitalized in nursing homes with logistical and organizational issues for daily and protracted access to radiotherapy departments or with reduced compliance.

Finally, the usefulness of this scheme could overwhelmingly be established in particular kinds of socio-health emergency contexts with the imperative need to limit access to health facilities and for time reduction of care for public health reasons (ie COVID-19 pandemic) [21, 24].

\section{Conclusions}

Hypofractionation of the radiotherapy dose for the treatment of elderly patients with NMSC of the face is safe and effective. The 35 Gy schedule in 5 fractions of 7 Gy delivered with low energy electron beam twice a week guarantees optimal patient collaboration and pursues a curative and functional result comparable to that obtainable with the standardized schedules. The unfitness of elderly and frail patients and the discomfort of the protracted schedules make randomized clinical trials difficult to conduct in order to compare between strongly hypofractionated $v s$. normofractioned schedules and, therefore, considering the emerging amount of data supporting hypofractionation, this option appears to be the most appropriate and valid choice in this specific patient setting. The most evident limitations of this experience are the sample size and the short duration of the follow-up, hence, studies with a much larger number of patients and with more mature data are to be encouraged.

\section{Conflict of interest}

The authors declare that they have no conflict of interest.

\section{Funding}

The authors received no specific funding for this work.

\section{References}

1. Wassberg C, Thörn M, Johansson AM, et al. Increasing incidence rates of squamous cell carcinoma of the skin in Sweden. Acta Derm Venereol. 2001; 81(4):
268-272, doi: 10.1080/00015550152572903, indexed in Pubmed: 11720174.

2. Goon PKC, Greenberg DC, Igali L, et al. Squamous Cell Carcinoma of the Skin has More Than Doubled Over the Last Decade in the UK. Acta Derm Venereol. 2016; 96(6): 820-821, doi: 10.2340/00015555-2310, indexed in Pubmed: 26631391.

3. Rogers HW, Weinstock MA, Feldman SR, et al. Incidence Estimate of Nonmelanoma Skin Cancer (Keratinocyte Carcinomas) in the U.S. Population, 2012. JAMA Dermatol. 2015; 151(10): 1081-1086, doi: 10.1001/jamadermatol.2015.1187, indexed in Pubmed: 25928283.

4. Boi S, Cristofolini M, Micciolo R, et al. Epidemiology of skin tumors: data from the cutaneous cancer registry in Trentino, Italy. J Cutan Med Surg. 2003; 7(4): 300-305, doi: 10.1007/s10227-002-0135-0, indexed in Pubmed: 12879331.

5. Busco S, Buzzoni C, Mallone S, et al. AIRTUM Working Group. Italian cancer figures - Report 2015: The burden of rare cancers in Italy. Epidemiol Prev. 2016; 40(1 Suppl 2): 1-120, doi: 10.19191/EP16.1S2.P001.035, indexed in Pubmed: 26951748.

6. Cognetta $A B$, Howard BM, Heaton HP, et al. Superficial $x$-ray in the treatment of basal and squamous cell carcinomas: a viable option in select patients. J Am Acad Dermatol. 2012; 67(6): 1235-1241, doi: 10.1016/j. jaad.2012.06.001, indexed in Pubmed: 22818756.

7. Park SY, Kang S, Park JM, et al. Development and dosimetric assessment of a patient-specific elastic skin applicator for high-dose-rate brachytherapy. Brachytherapy. 2019; 18(2): 224-232, doi: 10.1016/j.brachy.2018.11.001, indexed in Pubmed: 30528742.

8. Skowronek J. Brachytherapy in the treatment of skin cancer: an overview. Postepy Dermatol Alergol. 2015; 32(5): 362-367, doi: 10.5114/pdia.2015.54746, indexed in Pubmed: 26759545.

9. Scholten AN, Griep C, Davelaar J, et al. [Electron beam irradiation is effective in the treatment of skin carcinomas; a comparison with superficial roentgen therapy]. Ned Tijdschr Geneeskd. 1996; 140(8): 428-431, indexed in Pubmed: 8720817.

10. Pashazadeh A, Boese A, Friebe M. Radiation therapy techniques in the treatment of skin cancer: an overview of the current status and outlook. J Dermatolog Treat. 2019; 30(8): 831-839, doi: 10.1080/09546634.2019.1573 310, indexed in Pubmed: 30703334.

11. van Hezewijk M, Creutzberg $C L$, Putter $H$, et al. Efficacy of a hypofractionated schedule in electron beam radiotherapy for epithelial skin cancer: Analysis of 434 cases. Radiother Oncol. 2010; 95(2): 245-249, doi: 10.1016/j. radonc.2010.02.024, indexed in Pubmed: 20334941.

12. Kwan W, Wilson D, Moravan V. Radiotherapy for locally advanced basal cell and squamous cell carcinomas of the skin. Int J Radiat Oncol Biol Phys. 2004; 60(2): 406-411, doi: 10.1016/j.ijrobp.2004.03.006, indexed in Pubmed: 15380573.

13. McPartlin AJ, Slevin NJ, Sykes AJ, et al. Radiotherapy treatment of non-melanoma skin cancer: a survey of current UK practice and commentary. Br J Radiol. 2014; 87(1043): 20140501, doi: 10.1259/bjr.20140501, indexed in Pubmed: 25189280.

14. Chan S, Dhadda AS, Swindell R. Single fraction radiotherapy for small superficial carcinoma of the skin. Clin 
Oncol (R Coll Radiol). 2007; 19(4): 256-259, doi: 10.1016/j. clon.2007.02.004, indexed in Pubmed: 17379488.

15. Orton CG, Ellis F. A simplification in the use of the NSD concept in practical radiotherapy. Br J Radiol. 1973; 46(547): 529-537, doi: 10.1259/0007-1285-46-547-529, indexed in Pubmed: 4718294

16. Silva JJ, Tsang RW, Panzarella T, et al. Results of radiotherapy for epithelial skin cancer of the pinna: the Princess Margaret Hospital experience, 1982-1993. Int J Radiat Oncol Biol Phys. 2000; 47(2): 451-459, doi: 10.1016/s03603016(00)00410-7, indexed in Pubmed: 10802373.

17. Gunaratne DA, Veness MJ. Efficacy of hypofractionated radiotherapy in patients with non-melanoma skin cancer: Results of a systematic review. J Med Imaging Radiat Oncol. 2018; 62(3): 401-411, doi: 10.1111/1754-9485.12718, indexed in Pubmed: 29524319.

18. Zaorsky NG, Lee CT, Zhang E, et al. Hypofractionated radiation therapy for basal and squamous cell skin cancer: A metaanalysis. Radiother Oncol.2017; 125(1): 13-20, doi: 10.1016/j. radonc.2017.08.011, indexed in Pubmed: 28843727.

19. Tsao MN, Tsang RW, Liu FF, et al. Radiotherapy management for squamous cell carcinoma of the nasal skin: the Princess Margaret Hospital experience. Int J Radiat Oncol Biol Phys. 2002; 52(4): 973-979, doi: 10.1016/s03603016(01)02752-3, indexed in Pubmed: 11958891.
20. Veness M. Hypofractionated radiotherapy in older patients with non-melanoma skin cancer: Less is better. Australas J Dermatol. 2018; 59(2): 124-127, doi: 10.1111/ ajd.12609, indexed in Pubmed: 28294289.

21. Simcock R, Thomas TV, Estes C, et al. COVID-19: Global radiation oncology's targeted response for pandemic preparedness. Clin Transl Radiat Oncol. 2020; 22: 55-68, doi: 10.1016/j.ctro.2020.03.009, indexed in Pubmed: 32274425.

22. Pelissero A, Russi EG, Melano A, et al. Facial basal cell carcinomas treated with hypo-fractionated radiotherapy: A retrospective analysis in 117 elderly patients. J Am Acad Dermatol. 2015; 73(1): 166-168, doi: 10.1016/j. jaad.2015.03.030, indexed in Pubmed: 26089054.

23. Russi EG, Pelissero A, Melano A, et al. Facial Basal Cell Carcinomas in Elderly Frail Patients Treated with Low Total-dose Radiotherapy. Anticancer Res. 2015; 35(9): 4949-4953, indexed in Pubmed: 26254393.

24. Jereczek-Fossa BA, Pepa M, Marvaso G, et al. AIRO (Italian Association of Radiotherapy and Clinical Oncology). COVID-19 outbreak and cancer radiotherapy disruption in Italy: Survey endorsed by the Italian Association of Radiotherapy and Clinical Oncology (AIRO). Radiother Oncol. 2020; 149: 89-93, doi: 10.1016/j.radonc.2020.04.061, indexed in Pubmed: 32413527. 